\title{
A eficiência das redes sociais em processos de recrutamento organizacional
}

\begin{abstract}
RESUMO
Os avanços tecnológicos têm promovido mudanças sociais e, consequentemente, afetado o modo de gerir pessoas nas organizações. Neste sentido, este estudo se propõe a analisar a percepção de recrutadores sobre a eficiência do uso de redes sociais no processo de recrutamento organizacional. O referencial teórico que fundamentou o desenvolvimento do objetivo da pesquisa abordou os temas: Gestão Estratégica de Recursos Humanos e recrutamento; e- $\mathrm{RH}$ e práticas virtuais de recrutamento. Para tanto, realizou-se um estudo de caso, exploratório e descritivo com abordagem quantitativa. O questionário foi respondido por 45 recrutadores de quatro diferentes linhas de negócio da empresa ManpowerGroup. Os achados evidenciaram que, na percepção dos recrutadores, as redes sociais são eficientes na busca e identificação de candidatos nos processos de recrutamento organizacional comparativamente ao uso de outras estratégias, pois permite a rápida identificação de candidatos com perfil aderente à vaga. Diante dos resultados encontrados, sugerem-se investigações que ampliem a amostra ao ponto de favorecer a investigação dos questionamentos levantados.
\end{abstract}

Palavras-chave: e-RH. Recrutamento de pessoas. Gestão de Recursos Humanos. Gestão de Pessoas.

Cecilia Nascimento Cassiano Especialista em Gestão Estratégica de Pessoas Fundação Instituto de Administração (FIA/USP) - Brasil ceciliacassin@gmail.com

Luciana Campos Lima Doutoranda em Administração Fundação Instituto de Administração (FIA/USP) - Brasil lucamposlima@hotmail.com

Tatiani dos Santos Zuppani

Doutoranda em Administração Fundação Instituto de Administração (FIA/USP) - Brasil tszuppani@gmail.com 


\section{INTRODUÇÃO}

Em 2000, Breaugh e Starke publicaram o artigo "Research on Employee Recruitment: So Many Studies, So Many Remaining Questions", no qual explicitam que apesar das pesquisas sobre o tema recrutamento ter aumentado dramaticamente nos últimos trinta anos, muitas delas possuem um tom pessimista (BREAUGH; STARKE, 2000). Isso porque ainda se compreende muito pouco sobre as atividades relacionadas a essa prática. Os autores sugerem que estudos futuros tratem da complexidade que envolve o processo, considerando as variáveis e os atores envolvidos.

Esta lacuna de conhecimento ganha novos contornos diante dos fenômenos macroambientais, especialmente os relacionados aos avanços tecnológicos que acarretaram transformações sociais (CASTELLS, 2007). Breaugh (2008) destaca como recomendação para futuras pesquisas o maior envolvimento da tecnologia no recrutamento organizacional. Isso porque esse fator impõe mudanças às organizações, principalmente no seu modo de recrutar, contratar e gerenciar pessoas (FRIEDMAN, 2005). A internet é uma das principais ferramentas dessa revolução tecnológica.

$\mathrm{Na}$ área de Gestão de Recursos Humanos (GRH), essas mudanças podem ser verificadas, por exemplo, pela adoção de redes sociais como meio de comunicação e acesso ao mercado de trabalho. Na década de 90, surge a primeira rede social, o Classmates.com, possibilitando a extensão da vida pessoal ao mundo virtual. Em 2003, surge o Linkedin e as redes sociais se estendem ao cenário profissional e se reconfiguram como um importante meio de networking (GABRIEL, 2010).

Desde seu surgimento, essas e outras redes sociais impressionam pelo seu crescimento. Segundo dados do Linkedln, no ano de 2013, o Brasil atingiu a marca de 15 milhões de usuários, tornando-se o terceiro maior país em atividade nessa rede. Outras redes sociais apresentam números ainda mais expressivos. Segundo o Semiocast, empresa de pesquisa francesa, em 2013, no Brasil, o Facebook alcançou mais de 70 milhões de usuários, enquanto o Twitter, mais de 40 milhões.

Tais dados sugerem não apenas uma mudança de comportamento, mas uma oportunidade de desenvolvimento e competitividade às organizações que souberem aproveitar as redes sociais como ferramentas de trabalho. Para a área de Gestão de Recursos Humanos, elas podem ser úteis na obtenção de dados de potencias talentos e na geração de eficiência no tempo gasto com o recrutamento e seleção de candidatos.

Ao mesmo tempo em que essa ferramenta virtual mostra-se como uma oportunidade, configurase como um desafio a ser investigado e operacionalizado. O desafio para os pesquisadores tem sido demonstrar como o processo de recrutamento e seleção por este meio apresenta valor estratégico às organizações. Quando o impacto deste processo nas organizações é investigado, geralmente é descrito em termos simplistas, que não captam o nível de sofisticação que o recrutamento e a seleção têm alcançado nos últimos anos (LIEVENS; CHAPMAN, 2009).

O e-recruitment já se configura como uma ferramenta amplamente utilizada no contexto organizacional, como constatou a pesquisa da Robert Half, em 2009, com 375 executivos de todo o Brasil (ROBERT HALF, 2009). Os resultados revelaram que mais de $90 \%$ dos entrevistados da média e alta gerência usam redes sociais ativamente como ferramenta de trabalho e de contato com amigos e conhecidos. Em outra pesquisa, a Jobvite (2013) constatou que $94 \%$ dos selecionadores usam ou planejam utilizar redes sociais no processo de recrutamento e $78 \%$ usam essa ferramenta na contratação.

Reconhecida a dimensão que as redes sociais têm nas organizações atuais e considerando que o conhecimento científico acerca delas é incipiente no que tange ao recrutamento, a problemática desse estudo recai sobre a falta de informações empíricas que efetivamente demonstrem a eficiência de tais ferramentas, deixando a desejar no que diz respeito à sua potencial contribuição estratégica para a gestão das pessoas. Diante dessa lacuna, o objetivo traçado neste estudo foi analisar a eficiência do uso de redes sociais no processo de recrutamento organizacional.

Para tanto, foram investigadas fontes, formas e tempo de uso de redes sociais em processos de recrutamento. Estes aspectos foram avaliados procurando identificar a relação entre recursos consumidos e resultados alcançados - eficiência; e o alcance dos objetivos propostos - eficácia (SOBRAL; PECI, 2013). 
Quanto maior a eficiência e a eficácia desse processo, mais a GRH tende a contribuir para a estratégia do negócio.

Esse aspecto denota uma das principais contribuições desse estudo, delineando aspectos que potencialmente contribuem para o alcance das metas estabelecidas organizacionalmente. A pesquisa também traz para o campo científico um aspecto atual, relevante à realidade das empresas e que é bastante discutido nas mídias de negócio. Agregando, de tal modo, mais conhecimento para a tomada de decisão dos profissionais da área de gestão de pessoas.

Para a operacionalização desse estudo, buscou-se a fundamentação teórica acerca dos temas: Gestão Estratégica de Recursos Humanos e recrutamento; e-RH e práticas virtuais de recrutamento. Eles promoveram a base para o desenho do método empírico empregado e apresentado na seção de metodologia. Esta foi seguida pelos capítulos de apresentação, análise e discussão dos resultados, nos quais, além de expor os achados da pesquisa, buscou-se examinar seus significados práticos às organizações e como se posicionam teoricamente.

\section{REFERENCIAL TEÓRICO}

Este capítulo estrutura-se por meio da apresentação das práticas de gestão de pessoas voltadas para a captação e seleção de profissionais, especialmente, no que tange as ferramentas de e-RH.

\subsection{O uso de práticas na Gestão Estratégica de Recursos Humanos (GERH)}

A Gestão Estratégica de Recursos Humanos advém da combinação das práticas da área e o processo estratégico da organização. Para tanto, políticas e práticas de gestão de pessoas devem estar alinhadas às metas da organização e contribuir para que os empregados efetivamente alcancem resultados superiores (DEMO et al., 2011).

Para que esse alinhamento ocorra, as práticas de GRH têm evoluído ao longo dos anos. Na década de 30, elas estavam relacionadas aos termos e condições que estabeleciam políticas formais para se gerenciar a força de trabalho. Ao longo do tempo, foram direcionadas para lidar com questões de fluxo de funcionários, desempenho, informação e do próprio trabalho dentro da organização (STOREY; WRIGHT; ULRICH, 2009). $\mathrm{Na}$ atualidade, com um cunho mais estratégico, todos esses aspectos trabalham na promoção dos objetivos estabelecidos organizacionalmente. Isso viabiliza, conforme reconhecido por Armstrong (2006), uma conexão entre as práticas de Gestão de Recursos Humanos e o desempenho organizacional.

Entretanto, uma das tarefas primárias e básicas da GRH continua sendo buscar e contratar talentos para as organizações por meio das práticas de Recrutamento e Seleção (STOREY; WRIGHT; ULRICH, 2009).

\subsubsection{A prática de recrutar profissionais}

Para muitas empresas, o recrutamento e a seleção se tornaram ferramentas essenciais para o alcance de uma direção estratégica, continuidade de inovação, crescimento no mercado e garantia da vantagem competitiva e da alta performance nos negócios. Isso porque atrair e selecionar funcionários significa identificar e adquirir conhecimentos, habilidades e competências requeridas para alavancar o desempenho organizacional (STOREY; WRIGHT; ULRICH, 2009).

Segundo Lacombe e Heilborn (2003, p. 244) "o recrutamento abrange o conjunto de práticas e processos usados para atrair candidatos para as vagas existentes ou potenciais". É importante notar que, embora muitas vezes referenciado em conjunto com o conceito de seleção, tratam-se de práticas distintas. Para Limongi-França e Arellano (2002), seleção é a escolha, dentre todos os candidatos recrutados, aquele mais adequado à organização.

Para Breaugh e Starke (2000) e Chapman et al. (2005), o recrutamento afeta tanto a quantidade quanto os perfis de pessoas que estão dispostas a se candidatar, ou aceitar, uma oportunidade de trabalho. $O$ recrutamento se torna estratégico quando os profissionais de gestão de pessoas respondem a cinco 
questões essenciais: (I) quem recrutar, (II) onde recrutar, (III) quais recursos usar, (IV) quando recrutar e (V) qual mensagem comunicar (ORLITZKY, 2007; BREAUGH; STARKE, 2000).

A vantagem competitiva dessa prática, segundo a Visão Baseada em Recursos (VBR), é alcançada quando o recrutamento é capaz de: gerar eficiência de custos à mão de obra e/ou ampliar a percepção dos clientes quanto aos produtos e serviços da empresa; identificar e explorar os talentos que são raros ao mercado de trabalho; fazer com que as práticas de recrutamento sejam difíceis de imitar; estabelecer uma estratégia de recrutamento inovadora e peculiar para a organização, tornando-a insubstituível; alinhar-se às demais práticas de Gestão de Recursos Humanos, tais como reconhecimento, seleção e desenvolvimento (ORLITZKY, 2007).

Como forma de garantir a contribuição ao processo de manutenção da vantagem competitiva do recrutamento de profissionais, Breaugh e Starke (2000) apontam que é preciso ter uma clara ideia de quais são os objetivos do recrutamento e estabelecem uma estrutura simplificada para este processo conforme mostrado na Figura 1.

Figura 1 - Etapas do processo de recrutamento organizacional
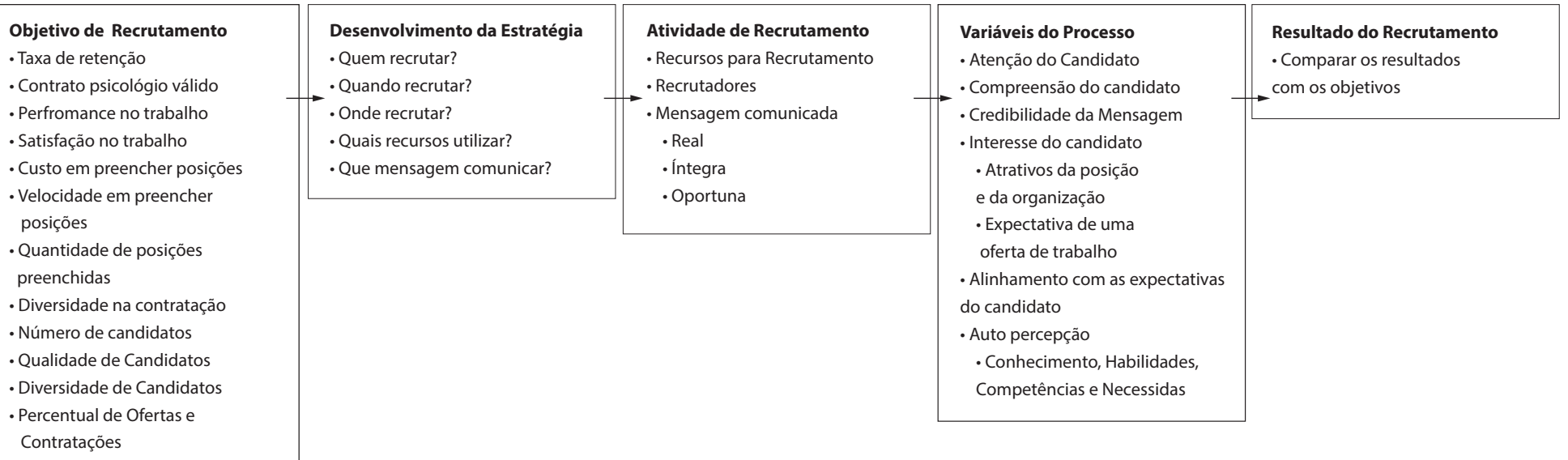

Fonte: Breaugh e Starke (2000, p. 408)

Por fim, é importante lembrar que o recrutamento é primeiro estágio na cadeia de valor da GRH. É ele quem controla e limita os demais processos subsequentes, como a seleção ou treinamento e desenvolvimento (ORLITZKY, 2007). Lievens e Chapman (2009) atestam que os candidatos constroem suposições em relação às organizações a partir do tratamento durante todo o processo seletivo. Para os autores, estas suposições podem influenciar diretamente seus níveis de interesse e atratividade.

\subsection{E-RH}

Com o advento do e-commerce, surge em 1990 o termo e-RH, que se refere à condução de processos de Gestão de Recursos Humanos com a utilização da tecnologia (LENGNICK-HALL; MORITZ, 2003). Segundo Nakayama, Pilla e Binotto (2006), o e-RH, ou, em inglês, e-HR (eletronic Human Resources) influencia processos de recrutamento e seleção com a utilização de recursos online, treinamentos a distância por meio de portais (e-learning), avaliações de desempenho conduzidas pela intranet ou por meio de softwares especializados, entre outros.

Em 1998, Ulrich antecipou-se à tendência constatando que o desenvolvimento futuro das práticas de GRH requer o contínuo aprimoramento de diversas tecnologias essenciais à área (ULRICH, 1998). Snell, Pedigo e Krawiec (1995) apud Lepak e Snell (1998) sugerem que a tecnologia da informação está integrada à área de gestão de pessoas de três diferentes formas: (i) pode atuar sob os aspectos operacionais da área, automatizando tarefas e práticas rotineiras; (ii) pode aperfeiçoar seu relacionamento com clientes internos e externos através da disponibilização de conteúdos relacionados aos processos e políticas, além de banco de dados e outras informações de esclarecimento, melhorando o relacionamento e a satisfação com a área e 
a empresa; e (iii) pode conectar a área ao seu papel transformacional, ao ampliar fronteiras geográficas e de tempo na transmissão de informações e na conexão entre equipes e espaços virtuais.

O uso da tecnologia do e-RH é um caminho para implantação de estratégicas da área de Gestão de Recursos Humanos, suas políticas e práticas (GUPTA; SAXENA, 2012) e para Lengnick-Hall e Moritz (2003) e tem potencial para influenciar tanto a efetividade quanto a eficiência dos processos. No que diz respeito à eficiência, provoca a redução de tempo de processamento e aperfeiçoa a checagem de informações, além de reduzir a quantidade de profissionais necessários para as atividades operacionais.

Da mesma forma que existem autores que defendem o e-RH, há aqueles que propõem a observação do uso de tecnologia na área com cautela, pois nem tudo pode ser resumido a benefícios. Huub, Bondarouk, Loois (2004) se propuseram a desmistificar o e- $\mathrm{RH}$, respondendo às seguintes questões: $\mathrm{O}$ que realmente é e-RH? Quais são os objetivos dele? Quais são os resultados que pode proporcionar?

Por meio da investigação de cinco organizações, os autores concluíram que o e-RH dificilmente ajudou a melhorar as competências dos funcionários, mas resultou em redução de custos e uma redução dos encargos administrativos.

\subsubsection{Processos Virtuais de Recrutamento}

No que diz respeito ao recrutamento, a tecnologia é considerada uma aliada, pois proporciona ferramentas eficazes para a captação de candidatos (NAKAYAMA; PILLA; BINOTTO, 2006). O recrutamento online permite o acesso a grande quantidade de informações de potenciais candidatos por um custo reduzido quando comparado às divulgações tradicionais em jornais e revistas (LIEVENS; CHAPMAN, 2009). Cappelli (2001) estima que o custo para se contratar um funcionário através da internet é vinte vezes menor que os custos de um processo em que não se utiliza a internet. Segundo o autor, as empresas ganham em eficiência com esse tipo de recrutamento: postagem de vagas online, currículos digitalizados e a busca eletrônica de perfis são formas de reduzir tempo e custo.

O processo de recrutamento online consiste na triagem, identificação e avaliação de candidatos através de ferramentas da internet. Os sistemas de recrutamento permitem armazenar currículos online e selecionálos por palavras-chave ou por outros critérios, como cargos e competências. Geralmente são adquiridos ou especialmente desenhados para as organizações a partir de fornecedores externos. Os sistemas transformam o currículo em papel em informações virtuais dos candidatos e possibilitam fácil acesso aos dados por todo o processo de recrutamento e seleção (GIRARD; FALLERY, 2010). Os sistemas de recrutamento combinados com os sites de empresas podem ser mais eficientes na busca do candidato, afirma Lee (2005). Múltiplos recursos de e-recruitment combinados em um mesmo processo aumentam a visibilidade da posição à candidatura de potenciais profissionais.

Neste contexto, quatro ferramentas se destacam nessa prática: sites de empresas, portais de carreira, job boards e, mais recentemente, redes sociais. Os job boards oferecem a possibilidade de divulgação de posições organizacionais para um grande público, com baixo custo e amplo acesso a currículos em banco de dados (GIRARD; FALLERY, 2010). São exemplos de job boards no Brasil o Vagas.com, InfoJobs e Catho.

O desenvolvimento dessas ferramentas virtuais para recrutamento e seleção de candidatos possibilita acesso em larga escala a uma importante base de informações de competências. Dessa forma, as organizações podem alcançar e gerenciar suas competências essenciais para obter vantagem competitiva no mercado, 0 que permite seu alinhamento aos conceitos da Visão Baseada em Recursos (GIRARD; FALLERY, 2010).

Tracey e Nathan (2002) apontam para uma nova forma de operacionalizar o recrutamento, que atribui à área de GRH o papel de desenvolver e executar o método de busca sugerido pelas demais áreas da empresa, elaborar um plano de comunicação com candidatos e apoiar e treinar gestores na condução de entrevistas e testes. A tecnologia contribui para que os gestores tenham autonomia na busca e identificação de perfis de candidatos através de diferentes ferramentas online.

A evolução tecnológica trouxe às mídias sociais novos meios de recrutamento, adotados como ferramentas, tais como blogs, plataformas de vídeo e redes sociais. Os blogs são espaços de compartilhamento de conteúdo, utilizados por candidatos e recrutadores para divulgação de vagas. Paralelamente surgiram 
os microblogs, dos quais o mais popular é o Twitter. Recrutadores se utilizam deles para publicar posições e realizar busca de perfis. Plataformas de vídeo (como Skype e Vimeo) permitem que empresas realizem a divulgação do seu trabalho e de posições abertas, enquanto candidatos introduzem seu currículo para análise.

\subsubsection{Mídias Sociais como estratégia de recrutamento e seleção}

Henderson e Bowley (2010) definem as mídias sociais como aplicações colaborativas online que permitem e incentivam o compartilhamento de informações entre os usuários, sendo elas: sites de redes sociais, wikis, blogs, podcasts, vidcasts (ou vlogs). Há quem as considera como sendo sistemas organizacionais complexos por envolverem um sistema de conhecimento compartilhado (HASGALL; SHOHAM, 2007).

A introdução da mobilidade, com o desenvolvimento dos smartphones e tablets, favoreceu o crescimento e popularidade das redes sociais (GABRIEL, 2010). A popularização dos sites de redes sociais proporcionou uma transmissão maior e em ritmo mais acelerado, por parte dos indivíduos, de informações sobre si mesmos e estas acabam sendo acessadas pelas empresas (HENDERSON; BOWLEY, 2010). Segundo Aguiar (2007), elas podem ser constituídas tanto espontaneamente, caracterizando as redes informais, quanto de forma intencional, o chamado network. Esse é estabelecido por indivíduos ou grupos que se articulam em torno de algo comum.

Redes sociais como Facebook e Google+ são plataformas de relacionamento social, enquanto que o Linkedin pode ser considerado uma plataforma especializada em networking profissional. Nestas redes é possível manter contato com amigos, colegas, profissionais, clientes e consumidores, além de estabelecer um diálogo com possíveis candidatos, futuros colaboradores e empregadores (GIRARD; FALLERY, 2010). O Facebook refere-se mais à esfera privada das pessoas. Apesar disso, tanto esse quanto o Linkedin permite a visibilidade de rede do indivíduo, o compartilhamento de conexões e a geração de capital social (HENDERSON; BOWLEY, 2010).

A divulgação da empresa via redes sociais é compreendida como um recrutamento passivo uma vez que a sua divulgação, de sua imagem e identidade, é realizada automaticamente, promovendo aos candidatos uma percepção mais acurada da personalidade da organização. Esta forma de recrutamento se desdobra por meio da divulgação da empresa pelos usuários de páginas de Facebook ou de grupo de fãs. Outro tipo de recrutamento passivo é a técnica de referências do Linkedin. Essas referências simplificam a coleta de informações sobre as qualificações dos candidatos e reduz os custos de recrutamento. Apesar de todas essas facilidades, deve-se considerar que o impacto das mídias sociais difere segundo as regiões, uma vez que dependem do acesso e disponibilização da internet (HENDERSON; BOWLEY, 2010).

De acordo com Qualman (2011), o Linkedin cresceu exponencialmente nos últimos anos; de $80 \%$ das empresas que utilizam mídias sociais para recrutamento, $95 \%$ usam essa rede social. No Brasil, o Linkedin e a Catho vêm desbancando o monopólio de formas mais tradicionais de divulgação de vagas, como os jornais (QUALMAN, 2011). Ainda segundo o autor, "usando ferramentas de mídias sociais durante o processo de recrutamento, as empresas têm uma chance melhor de manter talentos porque é mais provável que consigam colocar a pessoa certa no cargo certo" (QUALMAN, 2011, p. 249).

Em um estudo realizado com 398 entrevistados pertencentes a diversos setores e de organizações de vários tamanhos, Caers e Castelyns (2010) evidenciaram que 85\% dos usuários do Linkedin o empregam para verificar informações sobre a carreira dos amigos e $58 \%$ se atualizarem sobre o desenvolvimento em outras organizações. Com relação ao recrutamento, $44 \%$ dos respondentes afirmam usar o Linkedin para procurar potenciais candidatos que se encaixam nas vagas da organização. Os achados ainda demonstram que tanto o Linkedin quanto o Facebook não são considerados adequados para a realização de recrutamento interno; apenas $5,9 \%$ e 3,3\%, respectivamente, de entrevistados os consideraram para tal finalidade.

A adesão a esse ferramental, conforme já mencionado, não se dá de mesma forma em todas as regiões do mundo e há indícios de que os fatores culturais afetam a intensidade e aceitação das mídias sociais como contribuidores do processo de recrutamento de pessoas. Schinzel (2014) constatou que Luxemburgo vai contra essa tendência. Os profissionais de gestão de pessoas são relutantes em relação às tecnologias 
de redes sociais digitais. Essa relutância parece estar relacionada com a pontuação mais alta em "fuga à incerteza" de Hofstede. E resta a dúvida, e no Brasil, como os profissionais estão lidando com essa ferramenta?

\section{METODOLOGIA}

A pesquisa empírica promovida nesse estudo é quantitativa, descritiva e exploratória (SAMPIERI; COLLADO; LUCIO, 2006; VERGARA, 2007) e buscou ampliar o conhecimento sobre a eficiência das redes sociais no recrutamento organizacional. Inicialmente realizou-se um levantamento bibliográfico em bases nacionais e internacionais do Scientific Eletronic Library Online (Scielo), Portal de Periódicos (CAPES), EBSCO Host, Pro-Quest e Euromonitor International. As palavras-chave pesquisadas foram: recrutamento e recrutamento virtual (recruitment e e-recruitment), e-RH (e-HR e virtual HR), redes sociais (social network), tecnologia e RH (HR technology) e Gestão de Recursos Humanos (Human Resource Management).

$\mathrm{Na}$ sequência foi realizado o levantamento dos documentos referentes ao caso estudado - o ManpowerGroup no Brasil - via protocolo de estudo de caso, composto por uma visão geral da organização, pelos procedimentos de campo e o questionário. Para Martins e Theóphilo (2009, p. 62) o estudo de caso é "uma investigação empírica que pesquisa fenômenos dentro do seu contexto, buscando apreender a totalidade de uma situação e, criativamente, descrever, compreender e interpretar a sua complexidade". A aplicação de um estudo de caso único justifica-se pela singularidade das características do mesmo, uma vez que o ManpowerGroup é reconhecido no Brasil pela sua expertise em recrutamento e seleção.

A pesquisa de campo contemplou o universo dos empregados do ManpowerGroup no Brasil, sendo a amostra não probabilística e típica, constituída por 45 participantes. A elegibilidade destes seguiram os critérios de acessibilidade (LAVILLE; DIONE, 1999), baseado na ocupação do cargo efetivo de recrutador ou função semelhante. A coleta de dados foi realizada por meio do questionário do tipo auto-administrado, composto por 38 perguntas fechadas distribuídas nas categorias: caracterização do respondente, processo de recrutamento e uso de redes sociais. O questionário foi submetido a um pré-teste com quatro respondentes dentro do perfil típico da amostra. O propósito era testar a ferramenta de coleta de dados (LAKATOS; MARCONI, 1992). Com o resultado do pré-teste foi possível aprimorar o questionário.

A coleta dos dados teve como etapas: (i) envio de uma apresentação da pesquisa por e-mail com explicações sobre sua finalidade; (ii) envio do questionário via link da plataforma de pesquisas Survey Monkey, acompanhado de um texto autoexplicativo sobre o preenchimento e a confidencialidade das informações; e (iii) envio de um e-mail lembrete para reforçar a importância do preenchimento do questionário. $O$ período de retorno das respostas foi de 30 de abril a 09 de maio de 2014, sendo o lembrete enviado via Survey Monkey em 07 de maio de 2014. Foram enviados, ao todo, 81 questionários, sendo 43 para a Unidade de Negócios 1, sete para a Unidade de Negócios 2, oito para a Unidade de Negócios 3 e 23 para a Unidade de Negócios 4. Retornaram 45 questionários preenchidos, conforme a distribuição do Tabela 1.

Tabela 1 - Número de participantes por Unidade de Negócios

\begin{tabular}{lcccc}
\hline & Enviados & $\%$ & Recebidos & $\%$ \\
\hline Negócio 1 & 43 & $53 \%$ & 19 & $42 \%$ \\
Negócio 2 & 7 & $09 \%$ & 06 & $13 \%$ \\
Negócio 3 & 8 & $10 \%$ & 05 & $12 \%$ \\
Negócio 4 & 23 & $28 \%$ & 15 & $33 \%$ \\
\hline Total & 81 & $100 \%$ & 45 & $100 \%$ \\
\hline
\end{tabular}

Fonte: Autores (2015)

Para o tratamento e análise de dados utilizaram-se métodos descritivos como forma de organizar, sumarizar e descrever o conjunto de dados oriundos das respostas dos questionários (MARTINS; THEÓPHILO, 2007). Por tratar-se de um estudo exploratório, não se buscou a realização de testes de significância de diferenças encontradas, mas sim, descrever a percepção dos recrutadores da empresa pesquisada. 
Posteriormente, partiu-se para a análise e interpretação dos dados, para isso operacionalizou-se o cotejo dos construtos tratados na fundamentação teórica com a caracterização do caso e dos dados empíricos oriundos do questionário.

\section{DISCUSSÃO DOS RESULTADOS: O CASO MANPOWERGROUP}

A ManpowerGroup é uma consultoria fornecedora de soluções e serviços de mão de obra que oferece recrutamento, seleção, treinamento, desenvolvimento, gerenciamento de carreira e outsourcing. Os serviços são fornecidos pelas marcas Manpower para soluções de recrutamento de mão de obra temporária e permanente; Experis para recursos especializados e soluções de mão de obra customizadas; Right Management para soluções de gestão de carreira e talentos; e Solutions para terceirização de áreas de recrutamento em larga escala.

A empresa está presente em mais de 80 países com 3.100 escritórios, sendo a maior empresa de recolocação e líder global no mercado. Possui como visão: "Liderar o setor na criação e entrega de Innovative Workforce Solutions para que os clientes sejam bem-sucedidos no mundo do trabalho em constante mudança". Como valores organizacionais declara o respeito com todos os interessados no negócio, o compartilhamento do conhecimento e a inovação.

O processo de recrutamento empregado pela empresa se dá de forma semelhante nas quatro linhas de negócio, embora cada linha trabalhe com diferentes perfis e posições (Quadro 1).

Quadro 1 - Linhas de Negócios e seus processos de Recrutamento

\begin{tabular}{|l|c|c|c|c|}
\hline Indicadores & Negócio 1 & Negócio 2 & Negócio 2 & Negócio 4 \\
\hline $\begin{array}{l}\text { Ano de Criação no } \\
\text { Brasil }\end{array}$ & 2000 & 2012 & 2012 & 2012 \\
\hline Perfil de Posições & $\begin{array}{c}\text { Diversas (Níveis } \\
\text { Técnicos e } \\
\text { Operacionais) }\end{array}$ & $\begin{array}{c}\text { Profissionais de TI } \\
\text { (Níveis Técnico e } \\
\text { Gerencial) }\end{array}$ & $\begin{array}{c}\text { Profissionais de TI } \\
\text { (Níveis Técnico e } \\
\text { Gerencial) }\end{array}$ & $\begin{array}{c}\text { Administrativo e } \\
\text { (Níveis Técnico, } \\
\text { Gerencial) }\end{array}$ \\
\hline $\begin{array}{l}\text { Perfil de } \\
\text { Remuneração das } \\
\text { Posições }\end{array}$ & $\begin{array}{c}\text { Salários de até R\$ } \\
5.000,00\end{array}$ & Indiferente & Indiferente & Indiferente \\
\hline $\begin{array}{l}\text { Tipo de Contrato } \\
\text { das Posições }\end{array}$ & Temporário & $\begin{array}{c}\text { Temporário e } \\
\text { Permanente }\end{array}$ & $\begin{array}{c}\text { Temporário e } \\
\text { Permanente }\end{array}$ & $\begin{array}{c}\text { Temporário e } \\
\text { Permanente }\end{array}$ \\
\hline $\begin{array}{l}\text { Quantidade de } \\
\text { Respondentes }\end{array}$ & 19 Recrutadores & 06 Recrutadores & 06 Recrutadores & 15 Recrutadores \\
\hline
\end{tabular}

Fonte: Autores (2015)

O processo se inicia com uma solicitação de vaga pelo cliente, que varia entre temporária ou permanente. Quando a solicitação é feita o recrutador identifica as informações-chave da vaga (cargo, descrição do trabalho, competências requeridas, datas previstas de início e fim do trabalho, local de trabalho, subordinação, carga horária e remuneração). A partir de então, o processo é iniciado com a divulgação da posição e a busca e identificação de potenciais candidatos.

O negócio 1 é o mais antigo, envolve as posições que possuem salários até $\mathrm{R} \$ 5.000,00$, não atua com a contratação de empregados efetivos e possui o maior número de contratações de alocados. Já o negócio 2 tem foco exclusivo em profissionais da área de tecnologia da informação, inclusive efetivos, e não limita a faixa salarial. Os negócios 3 e 4 envolvem posições gerenciais e tratam de efetivos (negócio 3) e temporários e efetivos (negócio 4). As diferenças situam-se no formato de trabalho do negócio 4, que é por projeto, na contratação de alocados e no volume de recrutadores comparativamente maior. 
Com relação ao perfil dos recrutadores, identificou-se que mais de metade dos respondentes (60\%) possuem entre 22 a 29 anos, 31,3\% entre 30 a 39 anos, 6,7\% acima de 40 anos e 2,2\% entre 18 a 21 anos. Em relação à formação acadêmica, $96 \%$ dos respondentes possuem formação superior completa, sendo que destes, $27 \%$ são pós-graduados. No que tange o tempo de empresa, $49 \%$ dos recrutadores estão há menos de um ano na empresa, $40 \%$ estão em até três anos e $11 \%$ estão há mais de três anos.

\subsection{Análise das posições por negócios}

Ao analisar cada negócio é possível identificar a distribuição do volume médio de posições mensais (Tabela 2).

Tabela 2 - Distribuição do volume médio de posições por mês de acordo com os Negócios

\begin{tabular}{lccccc}
\hline & $\begin{array}{c}\text { Até } \\
10 \text { vagas }\end{array}$ & $\begin{array}{c}\text { De 11 a 20 } \\
\text { vagas }\end{array}$ & $\begin{array}{c}\text { De 21 a 30 } \\
\text { vagas }\end{array}$ & $\begin{array}{c}\text { De 31 a 40 } \\
\text { vagas }\end{array}$ & $\begin{array}{c}\text { Mais de 40 } \\
\text { vagas }\end{array}$ \\
\hline Negócio 1 & $21,05 \%$ & $5,26 \%$ & $21,05 \%$ & $26,32 \%$ & $26,32 \%$ \\
Negócio 2 & $16,67 \%$ & $83,33 \%$ & 0 & 0 & 0 \\
Negócio 3 & $80,00 \%$ & $20,00 \%$ & 0 & 0 & 0 \\
Negócio 4 & $13,33 \%$ & $40,00 \%$ & $20,00 \%$ & $6,67 \%$ & $20,00 \%$ \\
\hline \multicolumn{5}{c}{ Fonte: Autores (2015) }
\end{tabular}

Na análise da Tabela 2 destaca-se que os negócios 1 (acima de 31 vagas/mês) e 4 (13,33\% dos recrutadores com até 10 vagas, $40 \%$ de 11 a 20 vagas, $20 \%$ de 21 a 30 vagas, 6,67\% de 31 e 40 , e $20 \%$ com mais de 40 vagas) são os que possuem mais posições a serem trabalhadas por mês, o que justifica o número de recrutadores alocados. Entretanto, acredita-se que o volume elevado de posições seja explicada por motivos opostos, enquanto o negócio 1 atua com vagas que exigem baixa qualificação, o negócio 4 é justamente o contrário. Já os negócios 2 e 3 trabalham com até 20 vagas no mês e tratam de cargos técnicos especializados e gerenciais. Percebe-se então, uma relação entre o perfil da posição (qualificada, altamente especializada, etc.) e o volume de demandas.

\subsection{0 uso de redes sociais nos processos seletivos}

A análise do total da amostra que utiliza redes sociais em processos seletivos por linhas de negócio demonstra que há profissionais que "frequentemente" (35,6\%) as utilizam; 28,8\% afirmaram que "sempre" e "às vezes" utilizam esse tipo de ferramenta, e 6,8\% que "nunca" e "raramente" as utilizam. Já a análise do uso de redes sociais segundo os tipos de negócios evidencia que os negócios 1 (5,3\%) e 4 (21,4\%) são os que menos se utilizam de redes sociais em processos seletivos, enquanto que no negócio 3 "sempre" (100\%) se utiliza, seguido pelo negócio 2 (66,7\%).

Acredita-se que a opção por sempre utilizar redes sociais em processos seletivos do negócio 3 está relacionada ao perfil do candidato buscado. Assim, seriam os profissionais de média e alta gerência dos níveis técnico, operacional e gerencial, que possuem salário acima de $R \$ 5.000,00$ e que buscam um vínculo de trabalho efetivo os candidatos que mais são acessíveis por meio desse tipo de ferramenta. Da mesma forma, o negócio 1 foi o único que apresentou a resposta "raramente". Esse diz respeito aos profissionais temporários e terceirizados de posições operacionais e técnicas. Essas evidências demonstram a existência de algum tipo de relação entre o perfil da posição com o uso de redes sociais.

Identificou-se que $89 \%$ dos recrutadores que "frequentemente" e "sempre" utilizam os sites de carreiras acessam Catho, Vagas.com e Infojobs para divulgação das vagas, e $96 \%$ para a busca de currículos. Esse achado corrobora a afirmação de Nakayama, Pilla e Binotto (2006), ou seja, a adesão de mais de $80 \%$ dos 
recrutadores a essas ferramentas demonstra que a tecnologia realmente está sendo considerada uma aliada no processo de busca e atração de candidatos. Em relação às redes sociais que são utilizadas nos processos seletivos verificou-se que $91 \%$ dos recrutadores possuem um perfil pessoal ativo no Facebook, 86,6\% no Linkedin, $60 \%$ no Instagram, 31\% no Youtube e $28,9 \%$ no Google+. Dos que utilizam tais ferramentas "às vezes, frequentemente e sempre", tem-se que as redes sociais utilizadas de forma mais significativa nos processos seletivos são o Linkedin (90,5\%) e o Facebook (76,2\%); enquanto que o Google+ e Twitter seguem em terceiro lugar com 9,5\% cada um.

Os motivos à adesão dos recrutadores às redes sociais são apresentados na Figura 2.

Figura 2 - Motivos que levam os recrutadores a utilização de redes sociais em processos seletivos

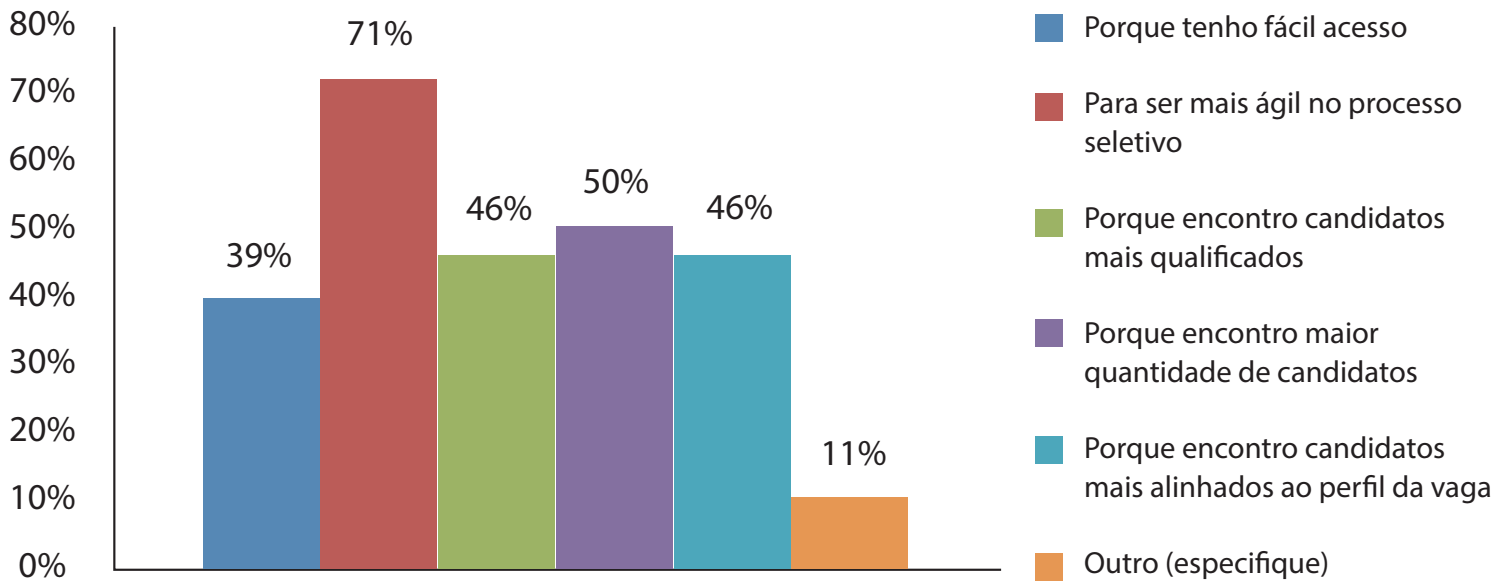

Fonte: Autores (2015)

A análise da Figura 2 permite identificar que 71,4\% dos respondentes acreditam que essa estratégia torna o processo mais ágil, $50 \%$ encontram maior quantidade de candidatos, e $46,4 \%$ consideram essa uma fonte de candidatos mais qualificados ao perfil da vaga. Dessa forma, o estudo demonstrou que, na percepção dos recrutadores que utilizam as redes sociais, estas são consideradas favoráveis aos processos de captura rápida de candidatos qualificados. Apesar de não ter investigado questões referentes ao custo do processo de recrutamento online, as empresas ganham em eficiência, uma vez que parecem reduzir o tempo de identificação dos potenciais candidatos (CAPELLI, 2001).

\subsubsection{Considerações sobre eficácia e eficiência dos processos de recrutamento}

Conforme demonstrado no item anterior, o aumento do uso de novas estratégias para divulgação e busca de candidatos por meio da internet está relacionada aos retornos percebidos pelos seus usuários. Com relação à divulgação de posições, os achados identificam que 28,9\% dos respondentes acreditam que o uso de site de carreira é o que oferece o melhor resultado. No que se refere à busca de currículos, 51,1\% deles consideram os sites de carreira como a fonte mais eficaz, e apenas 15,6\% consideram as redes sociais como as mais eficazes.

A análise comparativa com relação ao tempo despendido na busca de currículos mediante o uso de redes sociais em processos seletivos de posições do nível operacional (Figura 3) permitiu identificar que 60\% dos que utilizam redes sociais encontram candidatos adequados ao perfil em menos de uma hora. Já os que não utilizam redes sociais $(66,7 \%)$ levam "de duas a cinco horas". Esses achados validam a argumentação de Lee (2005) sobre a funcionalidade dos recursos e-recruitment, já que esses aumentam a visibilidade da posição e, portanto, podem aumentar a eficiência no processo. 
Figura 3 - Tempo de recrutamento de uma posição operacional

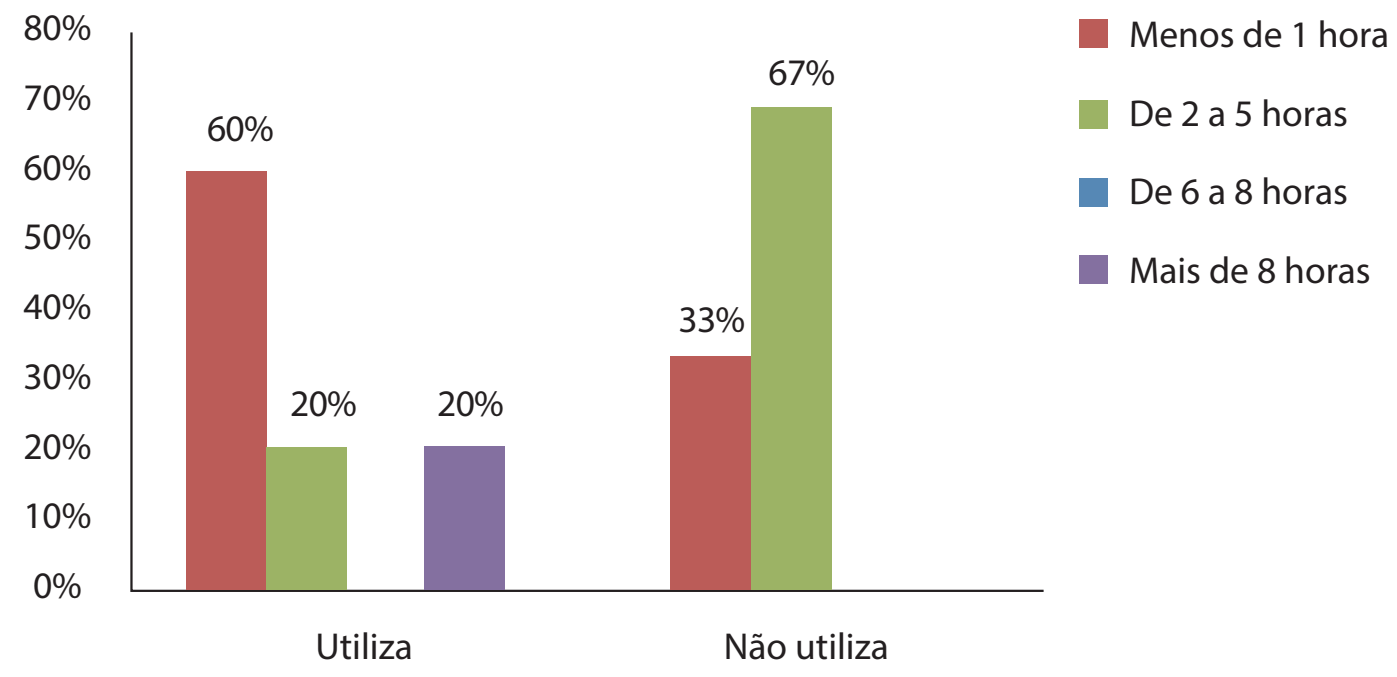

Fonte: Autores (2015)

Para as posições de nível técnico, $18,8 \%$ dos respondentes que não utilizam as redes sociais levam menos de uma hora para identificar um candidato, enquanto que apenas $14,8 \%$ dos que utilizam redes sociais encontram candidatos dentro desse período de tempo. Dos recrutamentos que não utilizam redes sociais, a maioria $(62,5 \%)$ leva entre duas e cinco horas para identificar os candidatos compatíveis com a vaga, sendo que $12,5 \%$ chegam a levar mais de oito horas. Já com relação aos que adotam essa ferramenta, $55 \%$ investem entre duas a cinco horas na identificação de perfis adequados e $29,6 \%$ de seis a oito horas (Figura 4).

Figura 4 - Tempo de recrutamento de um perfil técnico

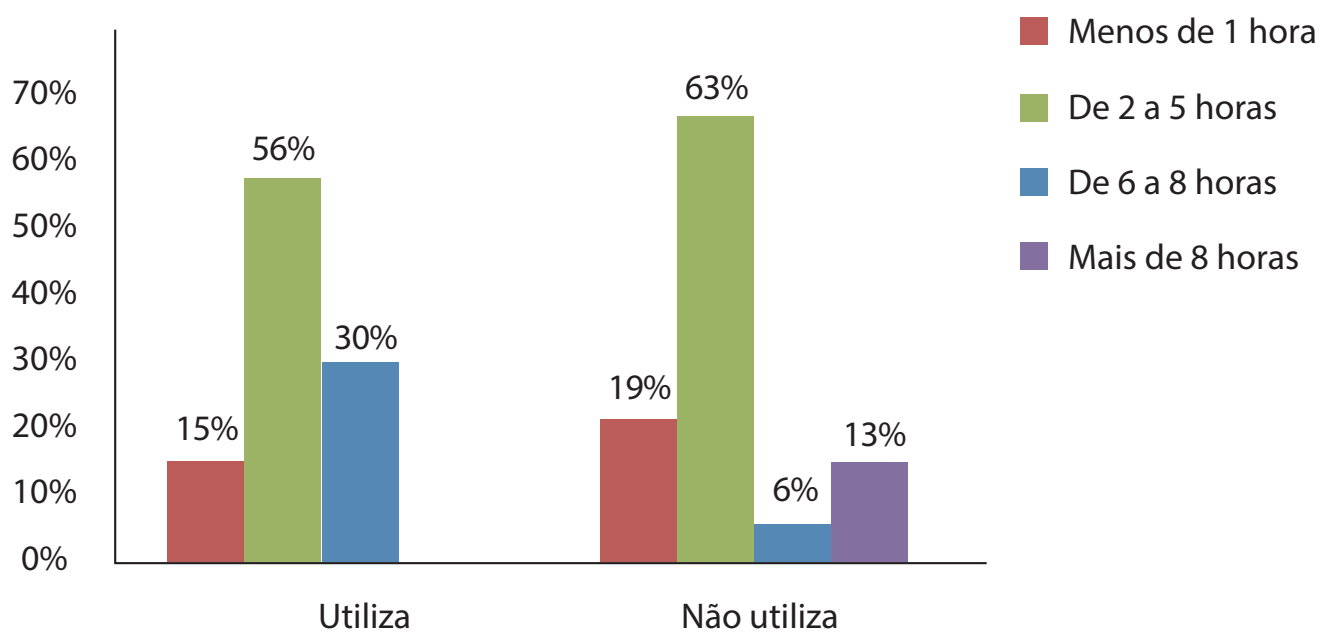

Fonte: Autores (2015)

Conforme figura 5, ao se tratar do nível administrativo, $18,5 \%$ dos respondentes que utilizam as redes sociais identificam os candidatos adequados em menos de uma hora, apenas $12,5 \%$ dos que não utilizam levam esse tempo. Uma grande maioria $(68,7 \%)$ dos que não utilizam redes sociais encontram os candidatos de "duas a cinco horas" e esse número cai para $63 \%$ no caso dos que usam. Permanece a busca por mais de oito horas $(12,5 \%)$ para essas vagas por parte dos que não utilizam a referida ferramenta. 
Figura 5 - Tempo de recrutamento de um perfil administrativo

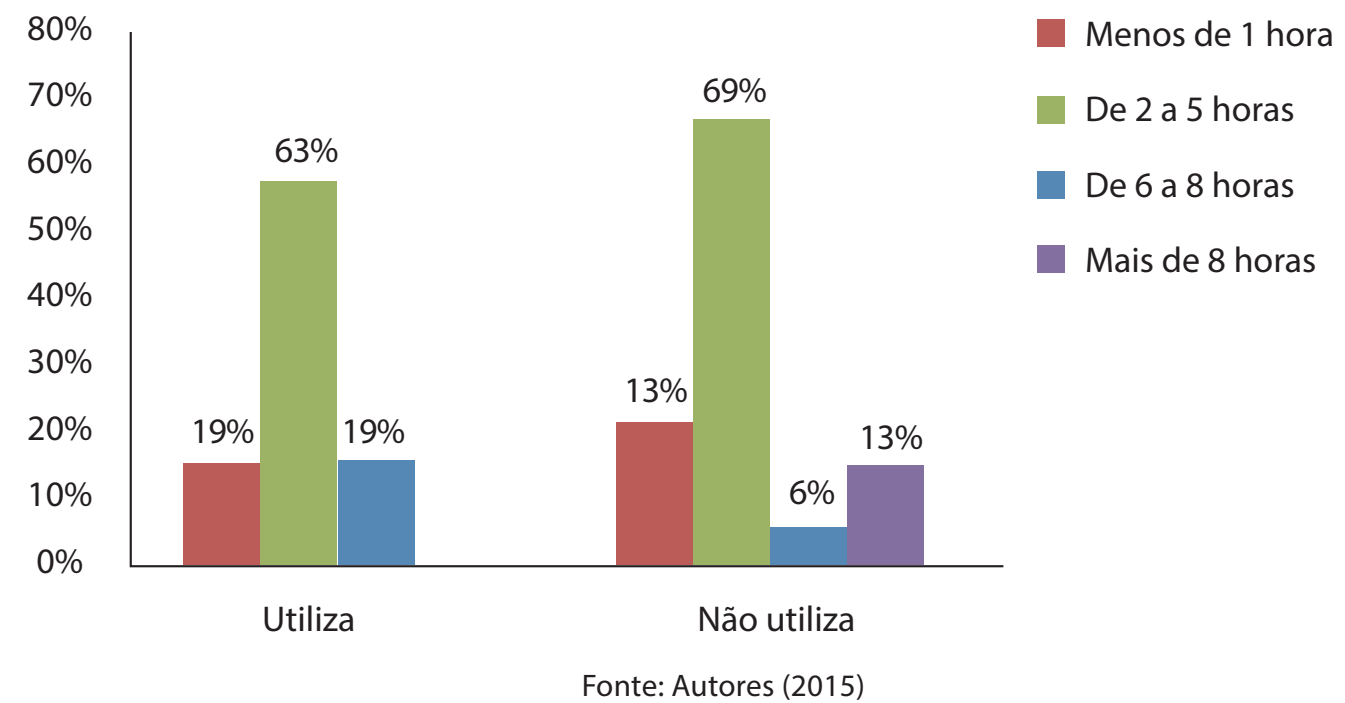

No caso das posições de nível gerencial destaca-se que os recrutadores que utilizam as redes sociais $(5,6 \%)$ encontram um candidato no nível gerencial em menos de uma hora. Isso não acontece no caso dos que não fazem uso dessa ferramenta. Com esses só será possível identificar um candidato a partir de duas horas de busca (28,5\%). Verificou-se um acréscimo de investimento em horas em ambos os casos, pois $38,9 \%$ dos que usam e $42,9 \%$ dos que não usam redes sociais buscam por mais de oito horas candidatos para as vagas (Figura 6).

Figura 6 - Tempo de fechamento de uma posição de perfil gerencial

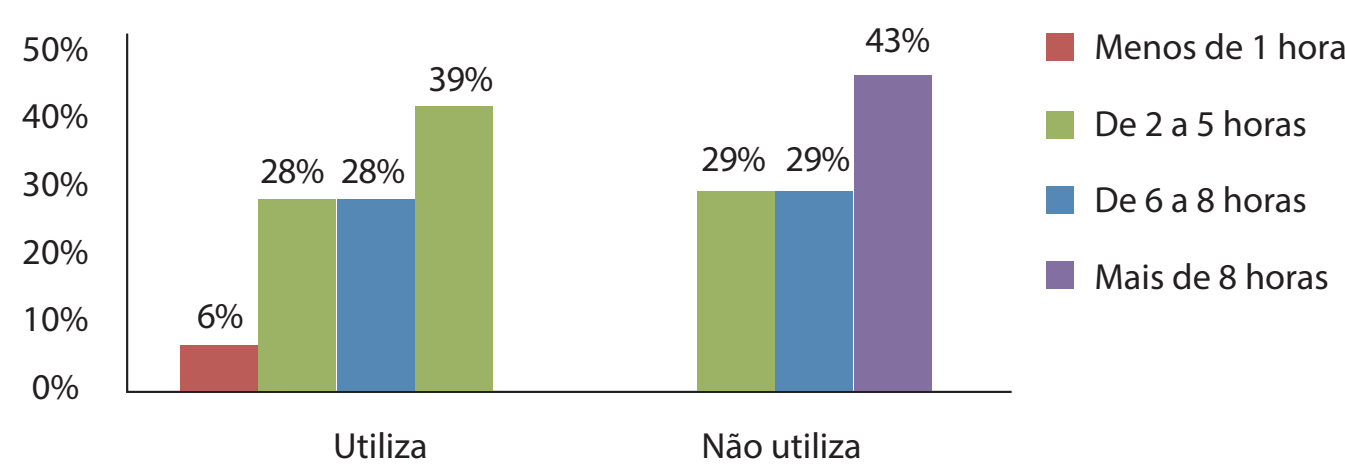

Fonte: Autores (2015)

Esses achados demonstram uma alta adesão por parte dos recrutadores ao uso das redes sociais como ferramenta para divulgação de posições e busca de candidatos $(93,4 \%)$. Dessa forma, infere-se que essa ferramenta está contribuindo para o que Girard e Fallery (2010) chamaram de "desenvolvimento de diálogos" entre os candidatos e seus futuros empregadores.

Apesar de apenas 5,3\% dos respondentes que atuam no negócio 1 utilizarem essa ferramenta, verificou-se que $60 \%$ das vagas operacionais possuem candidatos identificados em menos de uma hora. No que tange as posições técnicas pertencentes mais aos negócios 2 e 3, verificou-se que $81,3 \%$ das vagas trabalhadas sem o uso de redes sociais levam até cinco horas para a identificação de potenciais candidatos, contra $70,7 \%$ dos que utilizam a ferramenta. Nesse caso, $12,5 \%$ das vagas chegam a levar mais de oito horas na identificação de candidatos. Nas posições gerenciais $5,6 \%$ dos potenciais candidatos são mapeados em menos de uma hora com o uso de redes sociais, entretanto, ao não adotar essa ferramenta, a identificação só acontece após duas horas. 
Essas evidências demonstram que, na percepção dos recrutadores, as redes sociais utilizadas no processo de recrutamento são mais eficazes comparativamente ao uso de outras estratégias. Isso porque, o seu uso permite a identificação de candidatos com perfil aderente às vagas de forma mais rápida. Esse achado vai ao encontro da afirmação de Lengnick-Hall e Moritz (2003), que afirmam que o e-RH potencializa a eficiência dos processos de recrutamento, uma vez que este reduz o tempo de busca e identificação dos candidatos.

\title{
5 CONSIDERAÇÕES FINAIS
}

A pesquisa realizada obteve dois tipos de achados importantes. O primeiro respondeu diretamente ao objetivo do estudo, ou seja, constatou-se que as redes sociais são eficientes na busca e identificação de candidatos nos processos de recrutamento na percepção dos recrutadores da empresa estudada, sendo este achado coerente com outros estudos apresentados no referencial teórico (GUPTA; SAXENA, 2012; LENGNICK-HALL; MORITZ, 2003; NAKAYAMA; PILLA; BINOTTO, 2006). Já o segundo trata de questionamentos surgidos a partir das análises dos dados. Esses dizem respeito a possíveis relações entre o perfil das posições e o volume de demanda, e também com a decisão de uso de tecnologias de e-recruitment.

Os questionamentos surgidos reforçam a necessidade de produção acadêmica que proporcione profundidade e ampliação de conhecimentos sobre o tema, especialmente com relação ao uso de tecnologia e seus efeitos sobre o recrutamento e retenção dos empregados. Como um estudo exploratório, acreditase ter cumprido com a proposta de analisar a percepção de profissionais que atuam diretamente com recrutamento dentro de um contexto de negócio de igual natureza. Diante de uma produção acadêmica bastante limitada que contemple a realidade brasileira e da impossibilidade de generalização dos achados desse estudo recomenda-se a criação de uma agenda de pesquisa que inclua questões do tipo: (i) a percepção dos recrutadores que pertencem às empresas de diferentes naturezas de negócio diferem dos resultados da empresa estudada? (ii) há diferença significativa entre as percepções dos recrutadores segundo a faixa etária? (iii) o que os recrutados pensam a respeito dos processos via mídias sociais? Além disso, também há a necessidade de estudos de abordagem qualitativa, que possam proporcionar diferentes perspectivas frente as respostas já obtidas.

Considera-se como a principal limitação do estudo o fato de um dos pesquisadores fazer parte do quadro de funcionários da consultoria. Como forma de impedir qualquer tipo de viés, buscou-se utilizar diferentes fontes de informações e a validação por parte dos demais pesquisadores dos dados coletados.

Um dos pontos que mais chamou a atenção da equipe de pesquisadores, que cabe o relato, diz respeito a pouca importância demonstrada ao tema no contexto brasileiro. Acredita-se que esse tema é de grande relevância, pois se envolve com a matéria-prima da competitividade do país. É sabido que profissionais qualificados, um mercado de trabalho fluido e boas alocações de competências fazem diferença no desempenho de uma nação. $O$ processo de recrutamento realiza a conexão entre esses três elementos.

\section{EFFICIENCY OF SOCIAL NETWORKS IN ORGANIZATIONAL RECRUITING PROCESSES}

\begin{abstract}
Technological advances have affected the Human Resource Management, including the organizational recruitment process. The theoretical framework that justified the development of the purpose of the study addressed the following topics: Strategic Human Resources Management and recruitment; e-HR and virtual recruitment practices. This research is a case study applying exploratory and descriptive methodology and with quantitative approach. 45 recruiters from four different areas of the company ManpowerGroup. answered the questionnaire. The findings indicate the efficiency of social networks in the search and identification of candidates in the recruitment process compared to the use of other strategies. Considering the results, we suggest investigations that broaden the sample and the adoption of the mixed approach in the futures studies.
\end{abstract}


Keywords: E-HR. Professional Recruitment. Human Resource Management. Personnel Management.

\section{REFERÊNCIAS}

AGUIAR, S. Redes sociais na internet: desafios à pesquisa. Intercom-Sociedade Brasileira de Estudos Interdisciplinares da Comunicação. In: Congresso Brasileiro de Ciências da Comunicação, 30., 2007, Santos. Anais... Santos: Universidade Católica de Santos, 2007.

ARMSTRONG, M. A Handbook of Human Resources Management Practice. 10. ed. United Kingdom: Kogan Page, 2006.

BREAUGH, J. A.; STARKE, M. Research on employee recruitment: so many studies, so many remaining questions. Journal of Management, v. 26, n. 3, p. 405-434, 2000.

BREAUGH, J. A. Employee recruitment: current knowledge and important areas for future research. Human Resource Management Review, v. 18, n. 3, p. 103-118, 2008.

CAPPELLI, P. Making the most of online recruiting. Havard Business Review, v. 79, n. 3, p. 139-146, 2001.

CAERS, R.; CASTELYNS, V. Linkedln and Facebook in Belgium: the influences and biases of social network sites in recruitment and selection procedures. Social Science Computer Review, v. 29 n. 4, p. 437-448, 2010.

CASTELLS, M. A Sociedade em Rede. São Paulo: Paz e Terra, 2007.

CHAPMAN, D. S. et al. Applicant attraction to organizations and job choice: a meta-analytic review of the correlates of recruiting outcomes. Journal of Applied Psychology, v. 90, n. 5, p. 928-944, 2005.

DEMO, G. et al. Políticas de gestão de pessoas no novo milênio: cenário dos estudos publicados nos periódicos da área de administração entre 2000 e 2010. Revista de Administração Mackenzie, v. 12, n. 5, p. 15-42, 2011.

FRIEDMAN, T. L. The World is Flat: a brief history of the twenty-first century. New York: Farrar, Straus and Giroux, 2005.

GABRIEL, M. Marketing na Era Digital: conceitos, plataformas e estratégias. São Paulo: Novatec, 2010.

GIRARD, A.; FALLERY, B. Human Resource Management on internet: new perspectives. The Journal Contemporary Management Research, v. 4, n. 2, p. 97-120, 2010.

GUPTA, A.; SAXENA, S. Electronic Human Resouce Management (e-HRM): growing role in organizations. Management Insight, v. 8, n. 1, p. 505-514, 2012.

HASGALL, A.; SHOHAM, S. Digital social network technology and the complex organizational systems. VINE, v. 37, n. 2, p. 180-191, 2007.

HENDERSON, A.; BOWLEY, R. Authentic dialogue? The role of "friendship" in a social media recruitment campaign. Journal of Communication Management, v. 14, n. 3, p. 237-257, 2010.

HUUB, R.; BONDAROUK, T.; LOOISE, J. K. E-HRM: innovation or irritation. An explorative empirical study in 
five large companies on web-based HRM. Management Revue, v. 15, n. 3, p. 364-380, 2004.

JOBVITE. Social Recruiting: survey results. 2013. Disponível em <http://web.jobvite.com/rs/jobvite/ images/Jobvite_2013_SocialRecruitingSurveyResults.pdf>. Acessado em: 1 jul. 2014.

LACOMBE, F. J. M.; HEILBORN, G. L. J. Administração: princípios e tendências. São Paulo: Saraiva, 2003.

LAKATOS, E. M.; MARCONI, M. A. Metodologia do Trabalho Científico. São Paulo: Atlas, 1992.

LAVILLE, C.; DIONNE J. A Construção do Saber: manual de metodologia da pesquisa em ciências humanas. Porto Alegre: ARTMED, 1999.

LEE, I. Evaluation of fortune 100 companies' career web sites. Human Systems Management, v. 24, p. 175$182,2005$.

LENGNICK-HALL, M.; MORITZ, S. The impact of e-HR on the Human Resource Management function. Journal of Labor Research, v. 24, n. 3, p. 365-379, 2003.

LEPAK, D. P.; SNELL, S. A. Virtual HR: strategic human resource management in the 21 st century. Human Resource Management Review, v. 8, n. 3, p. 215-234, 1998.

LIEVENS, F.; CHAPMAN, D. Recruitment and selection. In: WILKINSON, A. et. al. The SAGE Handbook of Human Resource Management. [S.I]: Sage Publications Ltd, 2009. p. 133-154.

LIMONGI-FRANÇA, A. C.; ARELLANO, E. B. Os processos de recrutamento e seleção. In: FLEURY, M. T. L. (Org.) As Pessoas na Organização. 11. ed. São Paulo: Gente, 2002. p. 61-71.

MARTINS, G. A.; THEÓPHILO, C. R. Metodologia da Investigação Científica para Ciências Sociais Aplicadas. 2. ed. São Paulo: Atlas, 2009.

NAKAYAMA, M. K.; PILLA, B. S.; BINOTTO, E. (Org.) @-RH Conceitos e Práticas de RH Eletrônico. Passo Fundo: Editora UPF, 2006.

ORLITZKY, M. Recruitment strategy. In: BOXALL, P.; PURCELL, J.; WRIGHT, P. M. (Org.). The Oxford Handbook of Human Resource Management. Oxford: Oxford University Press, 2007. p. 273- 299.

QUALMAN, E. Socialnomics: How social media transforms the way we live and do business. 2. ed. New Jersey: Jonh Wiley \& Sons, 2011.

ROBERT HALF. Pesquisa da Robert Half aponta que mais de $90 \%$ dos executivos usam mídias sociais. 2009. Disponível em < http://www.roberthalf.com.br/portal/site/rh- br/menuitem.b0a52206b89cee97e7df ed10c3809fa0/?vgnextoid=d6ce296ddd375210VgnVCM 1000003c08f90aRCRD > Acessado em: 1 jul. 2014.

SAMPIERI, R. H.; COLLADO, C. F.; LUCIO, P. L. Metodologia de Pesquisa. 3. ed. São Paulo: McGraw Hill, 2006.

SOBRAL, F; PECl, A. Administração: teoria e prática no contexto brasileiro. 2. ed. São Paulo: Pearson, 2013.

SCHINZEL, U. Who Wants digital HRM? The example of Luxembourg. Journal of Business and Economics, v. 5, n. 12, p. 2374-2390, 2014. 
STOREY, J.; ULRICH, D.; WRIGHT, P. M. Introduction. In: STOREY, J.; ULRICH, D.; WRIGHT, P. M. (org.) The Routledge Companion to Strategic Human Resource Management. Routledge: Taylor \& Francis, 2009. p. 3-13.

TRACEY, J. B.; NATHAN, A. E. The strategic and operational roles of HR: a new model emerges. Cornell Hotel and Restaurant Admistration Quarterly, n. 43, p. 17-26, 2002.

ULRICH, D. Os Campeões de RH: inovando para obter os melhores resultados. São Paulo: Futura, 1998.

VERGARA, S. C. Projetos e Relatórios de Pesquisa em Administração. 8. ed. São Paulo: Atlas, 2007. 